\title{
FREQUENCY AND RISK FACTORS OF LEFT VENTRICULAR GEOMETRIC ABNORMALITIES IN HYPERTENSIVE PATIENTS: A STUDY BASED ON THE UPDATED CLASSIFICATION SYSTEM OF LEFT VENTRICULAR GEOMETRY
}

\author{
Fauzia Nazir, Tahir Iqbal, Javeria Kamran, Tariq Hussain Khattak, Anum Fatima, Aatika Habib, Saleh Kaleem \\ Armed Forces Institute of Cardiology/National Institute of Heart Disease (AFIC/NIHD)/National University of Medical Sciences (NUMS) \\ Rawalpindi Pakistan
}

\begin{abstract}
Objective: To determine the association between cardiovascular risk factors and the abnormalities of left ventricular geometric abnormalities.

Study Design: Prospective cross-sectional, single centered study.

Place and Duration of Study: Armed Forces Institute of Cardiology, Rawalpindi, from Jun 2018 to Dec 2018.

Methodology: This study permission was sought from hospital ethics committee. Written informed consent was taken from participants of study. Particulars of all the patients who meet the inclusion were included i.e., 351 hypertensive.

Results: Left ventricular geometric abnormalities were detected in 321 subjects (91\%), wherein concentric non dilated left ventricular hypertrophy is the most common left ventricular geometric abnormality (39\%). Elevated systolic blood pressure and diabetes mellitus were positively associated with concentric left ventricular remodeling, whereas body mass index and chronic kidney disease were inversely associated with concentric abnormalities. systolic blood pressure and diabetes mellitus, chronic kidney disease, large WC were positively associated with eccentric dilated left ventricular hypertrophy, while body mass index, duration of hypertension, MS were inversely associated with eccentric dilated left ventricular hypertrophy. Elevated systolic blood pressure was the strongest risk factor for eccentric dilated left ventricular hypertrophy. Large WC, systolic blood pressure and diabetes mellitus were positively associated with concentric left ventricular hypertrophy, whereas body mass index was negatively associated with concentric left ventricular hypertrophy.

Conclusion: Appropriate risk factor management and compliance can prevent left ventricular geometric abnormalities hence poorer outcomes in our population
\end{abstract}

Keywords: Cardiovascular risk factor, Hypertension, Left ventricular geometric abnormality, Left ventricular remodeling.

\footnotetext{
This is an Open Access article distributed under the terms of the Creative Commons Attribution License (http://creativecommons.org/licenses/by/4.0), which permits unrestricted use, distribution, and reproduction in any medium, provided the original work is properly cited.
}

\section{INTRODUCTION}

Hypertension and hypertensive heart disease continue to receive research interests because 30$45 \%$ of the general population are hypertensive and are often not or insufficiently treated and because issues such as the best treatment strategies and therapeutic goals remain indefinite ${ }^{1}$. Hemodynamic load caused by arterial hypertension may alter left ventricular (LV) function (systolic and diastolic) and cause ventricular remodeling (changes in size, shape, structure, and function) of the heart as a compensatory mechanism of increased wall stress and after load ${ }^{2}$.

Correspondence: Dr Fauzia Nazir, Department of Cardiology, AFIC/NIHD, Rawalpindi Pakistan
Left ventricular hypertrophy $(\mathrm{LVH})$ is a wellknown adaptive phenomenon, most commonly a result of untreated or uncontrolled hypertension. Numerous studies have shown that LVH is a strong and independent predictor of cardiovascular morbidity and mortality. Both combined or mixed hypertension and isolated systolic hypertension can result in various degrees of LVH. Different geometric patterns of hypertensive LVH were first described by Ganau ${ }^{3}$. It was later shown that different types of LV geometrical adaptations were associated with different hemodynamic patterns.

Recently, the investigators of the Dallas Heart study suggested a new classification for $\mathrm{LVH}$ based on four subtypes: eccentric non- 
dilated and dilated $\mathrm{LVH}$, and concentric nondilated and dilated LVH. The classification of LV geometry had been updated by the American Society of Echocardiography and the European Association of Cardiovascular Imaging in 20154. Therefore, this study is designed to investigate the associations between different cardio metabolic risk factors and the different phenotypes of LV geometric abnormality based on the updated classification system.

\section{METHODOLOGY}

This was a prospective cross-sectional single centered study conducted in Armed Forces Institute of Cardiology using consecutive sampling for the duration of 6 months (1st June, 2018 to 1st December, 2018).

The objective of study was to determine the frequency and risk factors of left ventricular geometric abnormalities in hypertensive patients and to establish an association between cardiometabolic risk factors and different LVH phenotypes patterns.

All those patients were included in the study who had HTN if they meet any of the following criteria i.e SBP $140 \mathrm{mmHg}$ or greater, DBP $90 \mathrm{~mm}$ $\mathrm{Hg}$ or greater, or Current use of antihypertensive medication. Patients with comorbids of valvular disease, Hypertrophic cardiomyopathy, severe liver, thyroid, neoplastic disease were excluded from the study.

Study was initiated after permission from ethics committee and research department. Baseline demographic information of the patient (age, sex, BSA, duration of hypertension) were taken. Informed consent was taken from each patient, ensuring confidentiality. A full medical history was collected. Blood and urine samples, a physical examination, a standard 12-lead electrocardiogram, an echocardiogram and three sphygmomanometric blood pressure (BP) measurements in the sitting position at the time of the first visit in hospital was performed.

Five comparison groups were created on the basis of the classification of the LV geometry: normal LV geometry, concentric remodeling, eccentric non-dilated $\mathrm{LVH}$, eccentric dilated LVH and concentric LVH (including concentric nondilated $\mathrm{LVH}$ and concentric dilated $\mathrm{LVH}$ ). The clinical characteristics of the five groups were presented as frequencies and percentages for categorical variables and as the mean and standard deviation (SD) for continuous variables. Univariate associations between all underlying risk factors and each type of LV geometric abnormality were calculated by logistic regression analysis. Step-wise logistic regression analysis was performed to identify independent risk factors for each type of abnormal LV geometry. Variables were reviewed for clinical significance before testing. Step-wise selection of risk factors after adjustment for age, sex, BMI and duration of hypertension use was performed sequentially, with a default value for inclusion set at $p<0.05$. SPSS software version 23 was used for all analyses.

\section{RESULTS}

The general clinical characteristics of the study patients are shown in table-I. The study population comprised 351 hypertensive patients (41.9\% women), aged $56 \pm 12$ years. LV geometric abnormalities were found in 322 subjects (91\%) distributed as follows: $20 \%$ with concentric LV remodeling, $45 \%$ with concentric LVH (wherein $39 \%$ were concentric non-dilated LVH and $17.6 \%$ were concentric dilated $\mathrm{LVH}), 4.8 \%$ with eccentric non-dilated $\mathrm{LVH}$ and $10.2 \%$ eccentric dilated $\mathrm{LVH}$, and $8.2 \%$ with no LV geometric abnormalities.

Patients with any type of LVH were older, showed lengthier durations of hypertension, had higher SBP, higher HDL-C, and a lower GFR and high uric acid levels compared to those with normal LV geometry. There were no significant differences in DBP and the incidences of MS, between the five patterns of LV geometry. There were no significant differences in the use of each class of anti-hypertensive medication among the five patterns of LV geometry.

After adjustment for age, sex, BMI, duration of hypertension, and, step-wise multiple logistic 
Frequency and Risk Factors of LV Geometric Abnormalities Pak Armed Forces Med J 2020; 70 (Suppl-4): S695-700

regression analysis showed that the more risk was the strongest risk factor for concentric and factors were associated with eccentric dilated eccentric LV remodeling.

Table-I: Mean and SD of risk factors in different LV geometric abnormalities.

\begin{tabular}{|c|c|c|c|c|c|c|c|c|}
\hline & & $\begin{array}{l}\text { Total } \\
\text { n ( } \%)\end{array}$ & $\begin{array}{c}\text { Normal LV } \\
\text { Geometry } \\
\text { n ( } \%)\end{array}$ & $\begin{array}{l}\text { Concentric } \\
\text { LV } \\
\text { Remodeling } \\
\text { n (\%) }\end{array}$ & $\begin{array}{c}\text { Eccentric } \\
\text { Non- } \\
\text { dialated } \\
\text { LVH n }(\%)\end{array}$ & $\begin{array}{c}\text { Eccentric } \\
\text { Dialated } \\
\text { LV } \\
n(\%)\end{array}$ & $\begin{array}{c}\text { Concentric } \\
\text { dilated } \\
\text { LVH } \\
\text { n ( } \%)\end{array}$ & $\begin{array}{c}\text { Concentric } \\
\text { non- } \\
\text { dialated } \\
\text { LVH n }(\%)\end{array}$ \\
\hline \multicolumn{2}{|l|}{ Total } & 351 & 29 & 70 & 17 & 36 & 62 & 137 \\
\hline \multicolumn{2}{|l|}{ Age } & $56 \pm 13$ & $56.55 \pm 13.92$ & $53.6 \pm 13.7$ & $56 \pm 13.2$ & $58 \pm 11$ & $55 \pm 14.9$ & $57 \pm 11.98$ \\
\hline \multirow{2}{*}{ Gender } & Male & $204(58.1)$ & $16(55.2)$ & $42(60)$ & $9(52.9)$ & $24(66.7)$ & $31(8.8)$ & 82 \\
\hline & Female & $147(41.9)$ & $13(44.8)$ & $28(40)$ & $8(47.1)$ & $12(33.3)$ & $31(8.8)$ & 55 \\
\hline \multicolumn{2}{|c|}{ Duration of HTN } & $4.9 \pm 5.1$ & $3.36 \pm 3.02$ & $2.7 \pm 2.4$ & $9.8 \pm 7.6$ & $6 \pm 5.56$ & $6 \pm 5.55$ & $4.9 \pm 5.1$ \\
\hline \multicolumn{2}{|l|}{ SBP } & $139 \pm 16.8$ & $140 \pm 15.28$ & $138 \pm 1803$ & $138 \pm 24.3$ & $137 \pm 10$ & $141 \pm 18$ & $138 \pm 16.36$ \\
\hline \multicolumn{2}{|l|}{ DBP } & $84 \pm 10.4$ & $85.7 \pm 6.54$ & $83 \pm 8.62$ & $80.6 \pm 7.5$ & $83 \pm 9.5$ & $84 \pm 11.8$ & $85 \pm 11.74$ \\
\hline \multicolumn{2}{|l|}{ FBG } & $106 \pm 27$ & $100.96 \pm 33.18$ & $102 \pm 36.82$ & $111 \pm 12.8$ & $110 \pm 14.6$ & $105 \pm 18.4$ & $108 \pm 27.71$ \\
\hline \multicolumn{2}{|l|}{ TC } & $176 \pm 33.8$ & $158 \pm 25.4$ & $173 \pm 45.01$ & $146 \pm 28.8$ & $168 \pm 0.00$ & $165 \pm 17.6$ & $192 \pm 35.10$ \\
\hline \multicolumn{2}{|l|}{ LDL-C } & $131 \pm 50.27$ & $178.7 \pm 55.2$ & $131.5 \pm 50.2$ & $125 \pm 33.21$ & $154 \pm 41$ & $128 \pm 34$ & $137 \pm 52.5$ \\
\hline \multicolumn{2}{|l|}{ HDL-C } & $39 \pm 12.1$ & $45.85 \pm 8.50$ & $34 \pm 11.51$ & $37 \pm 11.3$ & $39 \pm 11.1$ & $41 \pm 12.9$ & $40 \pm 12.36$ \\
\hline \multicolumn{2}{|l|}{ GFR } & $102 \pm 42.64$ & $131 \pm 28.25$ & $104 \pm 36.5$ & $117 \pm 73$ & $88 \pm 36.6$ & $86.5 \pm 40.05$ & $102 \pm 42.84$ \\
\hline
\end{tabular}

LVH. Diabetes and elevated SBP were positively associated with both concentric and eccentric LV
Diabetes also had positive association in all other LV geometric abnormalities.

Table-II: Uni-variate logistic regression analysis to select risk factors associated with each phenotype of left ventricular geometric abnormality.

\begin{tabular}{|c|c|c|c|c|c|c|c|c|c|c|c|c|}
\hline \multirow[t]{2}{*}{ Variable } & \multicolumn{3}{|c|}{ Concentric Remodling } & \multicolumn{3}{|c|}{$\begin{array}{c}\text { Ecentric Non-Dilated } \\
\text { LVH }\end{array}$} & \multicolumn{3}{|c|}{ Ecentric Dilated LVH } & \multicolumn{3}{|c|}{ Concentric LVH } \\
\hline & OR & $(95 \% \mathrm{CI})$ & $p$ & OR & $(95 \% \mathrm{CI})$ & $p$ & OR & $(95 \% \mathrm{CI})$ & $p$ & OR & $(95 \% \mathrm{CI})$ & $p$ \\
\hline $\begin{array}{l}\text { Age, } \\
\text { years }\end{array}$ & .980 & $.947 \sim 1.01$ & .261 & .98 & $.94 \sim 1.0$ & .51 & 1.00 & $.96 \sim 1.03$ & .94 & .99 & $.96-1.02$ & .56 \\
\hline $\begin{array}{l}\text { Gender, } \\
\mathrm{F}, \mathrm{M}\end{array}$ & 1.37 & $.49 \sim 3.79$ & .543 & .86 & $.20 \sim 3.64$ & .839 & 2.075 & $.66 \sim 6.33$ & .21 & 1.47 & $.62 \sim 3.46$ & .37 \\
\hline BMI & .739 & $.42 \sim 1.2$ & .278 & .45 & $.23 \sim .86$ & $.016^{*}$ & .79 & $.42 \sim 1.48$ & .47 & .60 & .36 .98 & $.04^{*}$ \\
\hline $\begin{array}{l}\text { Duration } \\
\text { of HTN }\end{array}$ & .742 & $.65 \sim .84$ & $.001^{*}$ & .98 & $.88 \sim 1.09$ & .748 & .89 & $.80 \sim .99$ & $.04^{*}$ & .88 & $.81 \sim .96$ & $\begin{array}{c}.007 \\
*\end{array}$ \\
\hline $\begin{array}{l}\text { Elevated } \\
\mathrm{BP}\end{array}$ & 4.5 & 1.60 12.71 & $.004^{*}$ & 4.01 & $.76 \sim 20.21$ & .09 & 5.79 & $1.66-20.17$ & $.006^{*}$ & 1.35 & $.57 \sim 3.16$ & .49 \\
\hline WC & 1.00 & $.96 \sim 1.05$ & .705 & .99 & $.91 \sim 1.07$ & .82 & .99 & $.94 \sim 1.04$ & .848 & 1.01 & $.98 \sim 1.05$ & .36 \\
\hline CKD & .64 & $.17 \sim 2.37$ & .509 & 1.06 & $.18 \sim 6.1$ & .94 & .53 & $.15 \sim 1.78$ & .30 & .82 & $.29 \sim 2.33$ & .72 \\
\hline MS & .02 & $.004 \sim .12$ & $.001^{*}$ & .13 & $.01 \sim 1.53$ & .10 & .04 & $.007 \sim .268$ & $.001^{*}$ & .26 & $.06 \sim 1.20$ & .08 \\
\hline Diabetes & .89 & $.21 \sim 3.32$ & .87 & .84 & $.15 \sim 4.68$ & .85 & .85 & $.24 \sim 3.05$ & .80 & 1.62 & $.22 \sim 1.78$ & .38 \\
\hline $\begin{array}{l}\text { High } \\
\text { FBG }\end{array}$ & 1.3 & $.60 \sim 2.9$ & .47 & 4.39 & $1.19-16.11$ & $.02^{*}$ & 2.28 & $.86 \sim 6.02$ & .09 & 2.08 & $1.01 \sim 4.28$ & $.04^{*}$ \\
\hline High TG & 9.46 & $2.41 \sim 37$ & $.001^{*}$ & .86 & $.11 \sim 6.72$ & .89 & 3.42 & $.84 \sim 13.90$ & .08 & 1.45 & $.43 \sim 4.89$ & .54 \\
\hline $\begin{array}{l}\text { Low } \\
\text { HDL-C }\end{array}$ & .68 & $.22 \sim 2.1$ & .51 & 1.40 & $.27 \sim 7.25$ & .68 & 1.05 & $.37 \sim 3.54$ & .933 & .70 & $.27 \sim 1.81$ & .46 \\
\hline $\begin{array}{l}\text { Large } \\
\text { WC }\end{array}$ & $\begin{array}{c}16.0 \\
7\end{array}$ & $3.3 \sim 78.12$ & $.001^{*}$ & $\begin{array}{c}13.2 \\
9\end{array}$ & $\begin{array}{c}1.29 \\
-136.52\end{array}$ & $.02 *$ & 2.63 & $.95-13.99$ & .25 & 2.81 & $\begin{array}{c}.76 \sim \\
10.42\end{array}$ & .12 \\
\hline $\begin{array}{l}\text { Hyperure } \\
\text { cemia }\end{array}$ & .174 & $.04 \sim .62$ & $.008^{*}$ & .05 & .01 .28 & $.001^{*}$ & .88 & $.22-3.51$ & .86 & .36 & $.12 \sim 1.06$ & .06 \\
\hline
\end{tabular}

remodeling types, whereas elevated SBP [odds ratio (OR) 5.79 (95\% CI, 1.66 20.17), p-value 0.006]
In concentric remodeling hyperuricemia, age, diabetes and elevated SBP had a positive association with significant $p$-value. However, 
BMI, duration of HTN and CKD, MS, and high TG were negatively associated with concentric remodeling.

In eccentric non dilated type of LV geometry elevated SBP, DM, hyperuricemia were positively associated whereas high FBG and BMI, large WC, CKD, were negatively associated. Elevated SBP was the strongest risk factor.

In eccentric dilated type of $\mathrm{LVH}$ elevated $\mathrm{SBP}$, large WC, CKD, DM were positively associated and elevated SBP was the strongest risk factor (OR 5.79, 95\% CI 1.66 20.17, p-value 0.006) where as duration of HTN and MS, BMI and hyperuricemia had negative association.

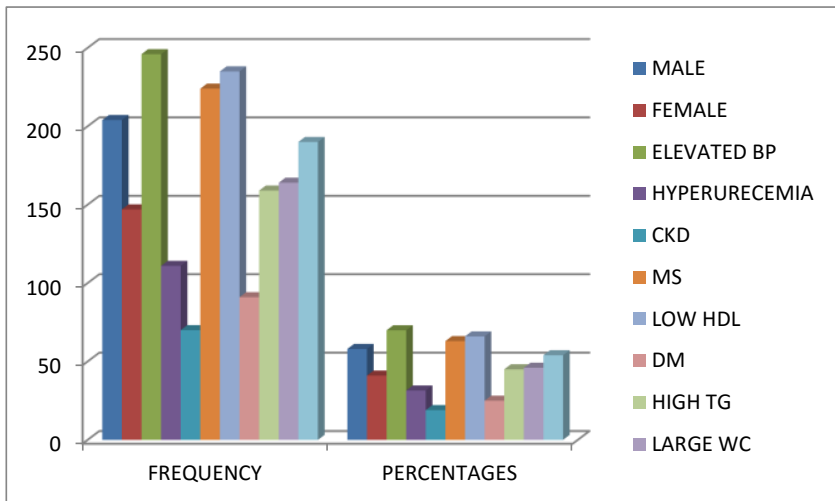

Figure: Distribution of risk factors in hypertensive population.

In concentric LVH elevated SBP, large WC, and diabetes were positively associated. Diabetes (OR 1.62, 95\% CI 0.22 1.78 P 0.041) turned out to be a strong risk factor for concentric $\mathrm{LVH}$, whereas BMI was negatively associated with concentric LVH.

Duration of HTN had a positive association with dilated types of LVH whereas BMI had negative association with dilated type of $\mathrm{LVH}$.

\section{DISCUSSION}

Hemodynamic load caused by arterial hypertension may alter left ventricular (LV) function (systolic and diastolic) and cause ventricular remodeling (changes in size, shape, structure, and function) of the heart as a compensatory mechanism of increased wall stress and after load.
Concentric nondilated LVH is associated with normal left ventricular chamber size, and left ventricular systolic function and performance comparable with that found in patients with normal left ventricular geometry, but substantially higher level of peripheral resistance and arterial stiffness, indicating a predominant pressure overload. In contrast, patterns of dilated $\mathrm{LVH}$ are characterized by dilated left atrium, greater left ventricular mass, enhanced left ventricular pump performance and normal-to-reduced peripheral resistance and arterial stiffness, suggesting a more prominent volume load component. The only remarkable difference between the two dilated left ventricular geometric patterns is that eccentric dilated $\mathrm{LVH}$ is associated with lower peripheral resistance and more predominant signs of volume overload than the concentric dilated type of $\mathrm{LVH}^{5}$. Eccentric dilated $\mathrm{LVH}$, concentric nondilated LVH and concentric dilated LVH were associated with higher cardiovascular risk 5 .

The present study displayed that the refined model adds prognostic information beyond simple measurement of LVM.

In a study by Okin et al approximately 5 years of antihypertension treatment greatly reduced the prevalence of both nondilated and dilated concentric $\mathrm{LVH}$, with a smaller reduction in eccentric dilated LVH demonstrating that hypertension treatment decreased the numbers in the 2 dilated groups. They demonstrated that 3 subtypes of LVH- eccentric dilated and both concentric patterns predict cardiovascular events, and that LVM regression has been shown to prevent cardiovascular morbidity and mortality 6 sufficient antihypertension treatment seems important to avoid the 3 high-risk subtypes of LVH: eccentric dilated and concentric nondilated and dilated LVH.

Sha et $a l^{7}$ reported the results of a retrospective analysis in patients with hypertension. They implemented a modified classification of geometric patterns first published by the Dallas Heart Study investigators. This new classification took into account the dimensions of the left 
ventricle and tabulated patients into six geometric patterns. Of the 2290 patients with hypertension, LV geometric abnormalities were noted in 1479 patients $(64.6 \%)$. They stated that concentric LV geometric abnormalities were more commonly accompanied by more cardiovascular risk factors such as increased waist circumference, neck circumference, old age, systolic BP, hyperuricemia, increased BMI, and alcohol use. ${ }^{7}$ In fact, these $\mathrm{CV}$ risk factors can partially explain the higher $\mathrm{CV}$ risk associated with concentric LV geometric patterns. However, this paper does not report outcomes associated with each geometric pattern, and it is difficult to create the link between the pattern of $\mathrm{LVH}$, risk factors, and the risk itself. Nevertheless, the assumption in this paper is that not only the geometric patterns but also the accompanied risk profile of the patient may be of importance.

In LIFE study, Watchtell et al specified that treatment and control of hypertension can dramatically alter these geometric patterns. In a group of 853 patients with ECG and echo-confirmed $\mathrm{LVH}$, treatment reduced the blood pressure from $174 / 95 \mathrm{mmHg}$ to $151 / 84 \mathrm{mmHg}$, and LV mass was reduced from 234 to $207 \mathrm{~g}$. Prevalence of concentric LVH decreased from $24 \%$ to $6 \%$, eccentric LVH decreased from $46 \%$ to $37 \%$, and concentric remodeling decreased from $10 \%$ to $6 \%$. Normal geometry increased from $20 \%$ to $51 \% .8-12$ Thus, effective treatment and control of hypertension is vital to change the geometric patterns that predict high cardiovascular risk.

Cuspidi et al studied the risk of cardiovascular and all-cause mortality associated with LV geometric patterns as defined by the new classification system proposed by the Dallas Heart study. A total of 1716 patients, contributors in the PAMELA study, were included. They reported that concentric LV remodeling was the most common geometric pattern $(9.4 \%)$, followed by eccentric non-dilated LVH (6.3\%). Compared to normal LV geometry, concentric LVH predicted cardiovascular risk of cardiovascular mortality by 4.04fold, dilated LVH by 3.83-fold, and eccentric nondilated LVH by 2.61-fold after adjustment for baseline covariates, including ambulatory blood pressure $^{13-18}$.

\section{CONFLICT OF INTEREST}

This study has no conflict of interest to be declared by any author.

\section{REFERENCES}

1. Mancia G, Fagard R, Narkiewicz K, Redón J, Zanchetti A, Böhm M, et al. 2013 ESH/ESC guidelines for the management of arterial hypertension: the Task Force for the Management of Arterial Hypertension of the European Society of Hypertension (ESH) and of the European Society of Cardiology (ESC). Eur Heart J 2013; 34(28): 2159-219.

2. Nadruz W. Myocardial remodeling in hypertension. J Hum Hypertens 2015; 29(1): 1-6.

3. Ganau A, Devereux RB, Roman MJ, de Simone G, Pickering TG, Saba PS, et al. Patterns of left ventricular hypertrophy and geometric remodeling in essential hypertension. J Am Coll Cardiol 1992; 19(7): 1550-58.

4. Khouri MG, Peshock RM, Ayers CR, de Lemos JA, Drazner MH. A 4-tiered classification of left ventricular hypertrophy based on left ventricular geometry: the Dallas heart study. Circ Cardiovasc Imaging 2010; 3(2): 164-71.

5. De Simone G, Izzo R, Aurigemma GP. Cardiovascular risk in relation to a new classification of hypertensive left ventricular geometric abnormalities. J Hypertens 2015; 33(4): 690-92.

6. Okin PM, Devereux RB, Harris KE, Jern S, Kjeldsen SE, Julius S, et al. LIFE Study Investigators. Regression of electrocardiographic left ventricular hypertrophy is associated with less hospitalization for heart failure in hypertensive patients. Ann Intern Med 2007; 147(5): 311-19.

7. Sha T, Huang YQ, Cai AP, Huang C, Zhang Y, Chen JY, et al. Prevalence and determinants of left ventricular geometric abnormalities in hypertensive patients: A study based on the updated classification system of left ventricular geometry. Hellenic J Cardiol 2017; 58(2): 124-32.

8. Wachtell K, Dahlo"f B, Rokkedal J. Change of left ventricular geometric pattern after 1 year of antihypertensive treatment: the losartan intervention for endpoint reduction in hypertension (LIFE) study. Am Heart J 2002; 144(6): 1057-64.

9. Cuspidi C, Facchetti R, Bombelli M. Risk of mortality in relation to an updated classification of left ventricular geometric patterns in a general population: The PAMELA study. J Hypertens 2015; 33(Suppl-1): e105.

10. Cramariuc D, Gerdts E. Epidemiology of left ventricular hypertrophy in hypertension: implications for the clinic. Expert Rev Cardiovasc Ther 2016; 14(8): 915-26.

11. Cuspidi C, Facchetti R, Bombelli M, Sala C, Tadic M, Grassi G, Mancia G. Risk of mortality in relation to an updated classification of left ventricular geometric abnormalities in a general population: the pamela study. J Hyperten 2015; 33(10): 2133-40.

12. Bang CN, Gerdts E, Aurigemma GP, Boman K, de Simone G, Dahlöf B, Køber L, Wachtell K, Devereux RB. Four-group classification of left ventricular hypertrophy based on ventricular concentricity and dilatation identifies a low-risk subset of eccentric hypertrophy in hypertensive patients. Circ Cardiovasc Imaging 2014; 7(3): 422-29.

13. Bang CN, Gerdts E, Aurigemma GP, Boman K, Dahlöf B, Roman MJ, et al. Systolic left ventricular function according to left ventricular concentricity and dilatation in hypertensive patients: the 
Frequency and Risk Factors of LV Geometric Abnormalities Pak Armed Forces Med J 2020; 70 (Suppl-4): S695-700

Losartan Intervention For Endpoint reduction in hypertension study. J Hyperten 2013; 31(10): 2060-68.

14. Kahan T, Persson H. The importance of left ventricular geometry in hypertensive heart disease. J Hyperten 2015; 33(4): 690-92.

15. Shenasa $M$, Shenasa $H$. Hypertension, left ventricular hypertrophy, and sudden cardiac death. Int J Cardiol 2017; 237: 60-63.

16. Erküner Ö, Dudink EAMP, Nieuwlaat R, Rienstra M, Van Gelder IC, Camm AJ, et al. Effect of systemic hypertension with versus without left ventricular hypertrophy on the progression of atrial fibrillation (from the Euro Heart Survey). Am J Cardiol
2018; 122(4): 578-83.

17. Nardi E, Palermo A, Mulè G, Cusimano P, Cerasola G, Rini GB. Prevalence and predictors of left ventricular hypertrophy in patients with hypertension and normal electrocardiogram. Eur J Prev Cardiol 2013; 20(5): 854-61.

18. Okin PM, Gerdts E, Wachtell K, Oikarinen L, Nieminen MS, Dahlöf B, et al. Relationship of left atrial enlargement to persistence or development of ECG left ventricular hypertrophy in hypertensive patients: implications for the development of new atrial fibrillation. J Hyperten 2010; 28(7): 1534-40. 